\title{
Structure of ${ }^{8} \mathrm{~B}$ and astrophysical $S_{17}$ factor in Skyrme Hartree-Fock theory
}

\author{
S.S. Chandel and S.K. Dhiman \\ Department of Physics, H.P. University, Shimla-171005, India \\ R. Shyam \\ Saha Institute of Nuclear Physics, AF/1, \\ Bidhan Nagar, Kolkata-700 064, India.
}

(Dated: October 31, 2018)

\begin{abstract}
We investigate the ground state structure of ${ }^{8} \mathrm{~B}$ within the Skyrme Hartree-Fock framework where spin-orbit part of the effective interaction is adjusted to reproduce the one-proton separation energy of this nucleus. Using same set of force parameters, binding energies and root mean square radii of other light p-shell unstable nuclei, ${ }^{8} \mathrm{Li},{ }^{7} \mathrm{~B},{ }^{7} \mathrm{Be}$, and ${ }^{9} \mathrm{C}$, have been calculated where a good agreement with corresponding experimental data is obtained. The overlap integral of ${ }^{8} \mathrm{~B}$ and ${ }^{7} \mathrm{Be}$ wave functions has been used to determine the root mean square radius of the single proton in a particular orbit and also the astrophysical $\mathrm{S}$ factor $\left(S_{17}\right)$ for the ${ }^{7} \mathrm{Be}(p, \gamma)^{8} \mathrm{~B}$ radiative capture reaction. It is found that the asymptotic region (distances beyond $4 \mathrm{fm}$ ) of the p-shell single proton wave function contributes more than half to the calculated value $(4.93 \mathrm{fm})$ of the corresponding single particle root mean square radius. The value of $S_{17}$ is determined to be $21.1 \mathrm{eV}$ b which is in good agreement with the recommended value for the near zero energy $S_{17}$ of $19.0_{-1.0}^{+4.0} \mathrm{eV}$ b.

PACS numbers: $21.60 . J_{z}, 21.10,21.65 .+f, 26.65 .+t$.
\end{abstract}




\section{INTRODUCTION}

Studies involving rare neutron rich and proton rich isotopes are currently at the forefront of the nuclear physics research. Experiments [1, 2] performed with beams of nuclei far from the beta stability (NFABS) line have revealed many features of these systems which are not present in their stable counterparts. For example, some NFABS having very small particle separation energies are very large in their radial dimensions (they are also called as halo nuclei); their radii are not governed by the usual $r_{0} A^{1 / 3}$ (with $\mathrm{r}_{0}=1.20$ and $\mathrm{A}$ the mass of the nucleus) rule. The discovery of these nuclei underlines the necessity of revising the traditional picture of nuclear structure in important ways since away from the $\beta$-stability nuclear dynamics are characterized by a variety of new features not present in stable nuclei. In the halo region the quantal dynamical effects play a crucial role in distribution of the nuclear density in the zone of very weak binding.

Proper knowledge of the structure of ${ }^{8} \mathrm{~B}$ nucleus is important for several reasons. This nucleus perhaps is the most likely candidate for having a proton halo structure, as its last proton has a binding energy of only $137 \mathrm{keV} .{ }^{8} \mathrm{~B}$ produced via ${ }^{7} \mathrm{Be}(\mathrm{p}, \gamma){ }^{8} \mathrm{~B}$ reaction in the Sun is the source of high energy neutrinos which are detected in SNO, Kamiokande and Homestake experiments [3, 4, 5]. Therefore, accurate determination of the cross section of this reaction at relative energies corresponding to solar temperatures (about $20 \mathrm{keV}$ ) is very important to the solar neutrino issue. In this energy region, the cross-section $\sigma_{p \gamma}\left(E_{c m}\right)$ [which is usually expressed in terms of the astrophysical $S_{17}\left(E_{c m}\right)$ factor] of the ${ }^{7} \mathrm{Be}(\mathrm{p}, \gamma)^{8} \mathrm{~B}$ capture reaction is directly proportional to the high energy solar neutrino flux. A better knowledge of $S_{17}$ is, therefore, important to improve the precision of the theoretical prediction of ${ }^{8} \mathrm{~B}$ neutrino flux from present and future solar neutrino experiments.

${ }^{7} \mathrm{Be}(\mathrm{p}, \gamma)^{8} \mathrm{~B}$ reaction has been studied extensively both theoretically as well as experimentally 6. 7, 8, 9, 10, 11, 12, 13, 14, 15, 16]. $S_{17}$ is determined either by direct measurements [17] or by indirect methods such as Coulomb dissociation [18] of ${ }^{8} \mathrm{~B}$ on heavy targets and transfer reactions in which ${ }^{8} \mathrm{~B}$ is either the residual nucleus or the projectile nucleus [19, 20, 21]. Efforts have also been made to calculate the cross section of this reaction within the framework of the shell model and the cluster model [23, 24, 25]. The key point of these calculations is the determination of the wave functions of ${ }^{8} \mathrm{~B}$ states within the given structure theory. 
Aim of the present study is to investigate the structure of ${ }^{8} \mathrm{~B}$ in the framework of the Skyrme Hartree-Fock (SkHF) model which has been used successfully to describe the groundstate properties of both stable [26, 27, 28] as well as exotic nuclei [29, 30, 31, 32]. The phenomena of nuclear skin and halo have been studied in medium mass and heavy nuclei 31, 32] within the Skyrme-Hartree-Fock-Bogoliubov method using the SLy4 Skyrme force. The SkHF method with density-dependent pairing correlation and SkI4 interaction parameters has been successful in reproducing the binding energies and rms radii [31] in the light neutron halo nuclei ${ }^{6} \mathrm{He},{ }^{8} \mathrm{He},{ }^{11} \mathrm{Li}$ and ${ }^{14} \mathrm{Be}$.

We solve axially symmetric Hartree-Fock (HF) equations with SLy4 [33] Skyrme interaction which has been constructed by fitting to the experimental data on radii and binding energies of symmetric and neutron-rich nuclei. This has also been used in Ref. [32] to study the phenomenon of shape coexistence in semi-magic isotopes of $\mathrm{Mg}, \mathrm{S}$ and $\mathrm{Zr}$ nuclei. In our calculations pairing correlations among nucleons have been treated within the BCS pairing method. We have, however, renormalized the parameter of the spin-orbit term of the SLy4 interaction so as to reproduce the experimental binding energy of the last proton in ${ }^{8} \mathrm{~B}$ nucleus. A check on our interaction parameters was made by calculating binding energies and $\mathrm{rms}$ radii of ${ }^{7} \mathrm{Be},{ }^{7} \mathrm{~B},{ }^{8} \mathrm{Li}$ and ${ }^{9} \mathrm{C}$ nuclei with the same set where a good agreement is obtained with corresponding experimental data. We calculate the root mean square radii for matter, neutron and proton distributions for ${ }^{8} \mathrm{~B}$. Using the overlap integral of HF wave functions for ${ }^{7} \mathrm{Be}$ and ${ }^{8} \mathrm{~B}$ ground states, the root mean square radius of the valence p-shell proton in this nucleus has been determined which is expected to provide information about the proton halo structure in ${ }^{8} \mathrm{~B}$. The overlap integral has also been used to calculate the astrophysical $S_{17}$ factor.

The paper is organized in the following way. In section II we present the short description of the method of our calculations. Section III contains our results and their discussions. Summary and conclusions of our study are given in section IV.

\section{METHOD OF CALCULATIONS}

In the Skyrme Hartree-Fock formalism, binding energies, densities and single particle wave functions are obtained from a local energy functional. Skyrme force parameters are determined empirically by fitting nuclear matter properties of stable nuclei and neutron star 
densities [33]. Microscopically, the Skyrme functional corresponds to an expansion of the nuclear interaction up to the first order in momentum transfer [26]. The results for ground state properties are derived self-consistently from the total energy functional of the nucleus which is given by

$$
\begin{array}{r}
E=E_{K E}+E_{s k}(\rho, \tau)+E_{\text {sk,ls }}(\rho, J)+E_{\text {Coul }}\left(\rho_{p}\right) \\
+E_{\text {Coul }, \text { exch }}\left(\rho_{p}\right)+E_{\text {pair }}-E_{C M}
\end{array}
$$

where $\rho_{p}$ is the proton density and

$$
\begin{gathered}
E_{K E}=4 \pi \sum_{q \in p, n} \int_{0}^{\infty} d r r^{2} \frac{\hbar}{2 m_{q}} \tau_{q}, \\
E_{S k}(\rho, \tau)=4 \pi \int_{0}^{\infty} d r r^{2}\left(\frac{b_{0}}{2 .} \rho^{2}+b_{1} \rho \tau-\frac{b_{2}}{2 .} \rho \nabla^{2} \rho+\frac{b_{3}}{3 .} \rho^{\beta+2 .}\right) \\
-4 \pi \sum_{q \in p, n} \int_{0}^{\infty} d r r^{2}\left(\frac{b_{0}^{\prime}}{2 .} \rho_{q}^{2}+b_{1}^{\prime} \rho_{q} \tau_{q}-\frac{b_{2}^{\prime}}{2 .} \rho_{q} \nabla^{2} \rho_{q}-\frac{b_{3}^{\prime}}{3 .} \rho^{\beta} \rho_{q}^{2}\right), \\
E_{S k, l s}(\rho, J)=-4 \pi \int_{0}^{\infty} d r r^{2}\left(b_{4} \rho \nabla J+b_{4}^{\prime} \rho_{q} \nabla J_{q}\right) .
\end{gathered}
$$

In above equations $q \in\{p, n\}$ is the isospin label for proton and neutron. In Eq. (4) an additional coefficient $b_{4}^{\prime}$ has been introduced in the spin-orbit term of the energy functional. The Coulomb energy term is given by

$$
E_{\text {Coul }}\left(\rho_{p}\right)=\frac{1}{2} e^{2} \int d^{3} r d^{3} r^{\prime} \rho_{C}(r) \frac{1 .}{\left|r-r^{\prime}\right|} \rho_{C}\left(r^{\prime}\right) .
$$

$E_{\text {Coul,exch }}$ is Coulomb exchange energy functional and it is usually treated in the Slater approximation,

$$
E_{\text {Coul }, \text { exch }}\left(\rho_{p}\right)=-\frac{3}{4}\left(\frac{3}{\pi}\right)^{1 / 3} 4 \pi \int_{0}^{\infty} d r r^{2} \rho_{p}^{\frac{4}{3}}(r) .
$$

The center of mass energy functional $E_{C M}$ is written as,

$$
E_{C M}=\frac{\left\langle P_{C M}^{2}\right\rangle}{2 A m}
$$

where $P_{C M}=\sum_{i} \hat{p}$ is the total angular momentum operator in the center of mass frame, which project out a state with good total angular momentum in the given mean-field state. We account for the pairing interaction among nucleons by solving BCS equations. The schematic pairing energy functional is given as

$$
E_{\text {pair }}=-\sum_{q \in p, n} G_{q}\left[\sum_{\alpha \in q} \sqrt{v_{\alpha}\left(1-v_{\alpha}\right)}\right]^{2}
$$


The pairing strength parameters are $G_{p}=29 / \mathrm{A} \mathrm{MeV} \mathrm{for} \mathrm{proton} \mathrm{and} G_{n}=29 / \mathrm{A} \mathrm{MeV}$ for neutron where $\mathrm{A}$ is number of nucleons in the nucleus. The occupation probabilities are given by

$$
v_{\alpha}^{2}=\frac{1}{2}\left[1-\frac{\left(\epsilon_{\alpha}-\epsilon_{F, \alpha}\right)^{2}}{\left(\epsilon_{\alpha}-\epsilon_{F, \alpha}\right)^{2}+\Delta_{q}^{2}}\right],
$$

where $\epsilon_{\alpha}$ is the single particle energy of the given orbit. The pairing gap $\Delta_{q}$ and the Fermi energy $\epsilon_{F, q}$ are obtained by the iterative solution of the gap equation and the particle number condition. Axially symmetric single particle wave functions for a nucleus are given in expansion basis by

$$
\psi_{\alpha}(r)=\phi_{\alpha}(r) \times Y_{l_{\alpha} j_{\alpha} m_{\alpha}}(\theta, \phi),
$$

where $\alpha$ represents the quantum numbers of the state $\mid n l j m>$ and spinor-spherical harmonics are given by $Y_{l_{\alpha} j_{\alpha} m_{\alpha}}(\theta, \phi)$. $\phi_{\alpha}(r)$ represents the radial wave function. Various phenomenological densities are used to construct the energy functional, particle densities $\rho_{q}(r)=\sum_{\alpha \in q} N_{\alpha}\left|\psi_{\alpha}(r)\right|^{2}$, kinetic densities $\tau_{q}(r)=\sum_{q \in q} N_{\alpha}\left|\nabla \psi_{\alpha}(r)\right|^{2}$, and spin-orbit densities $\nabla J_{q}(r)=-i \sum_{\alpha \in q} N_{\alpha} \nabla \psi_{\alpha}(r)^{+} \cdot \nabla \times \sigma \psi_{\alpha}(r)$. Usual total isoscalar densities without an isospin label are defined as $\rho=\rho_{\pi}+\rho_{\nu}, \tau=\tau_{\pi}+\tau_{\nu}$ and $J=J_{\pi}+J_{\nu}$, with summations being carried out over both proton and neutron particle numbers. $N_{\alpha}$ represents the desired proton or neutron number and is equal to $v_{\alpha}^{2}$ which is the BCS occupation probabilities of orbitals.

\section{RESULTS AND DISCUSSIONS}

\section{A. Binding energies, radii and density distributions}

The values of various parameters of the SLy4 Skyrme effective interaction as used in our calculations are: $t_{0}=-2488.91, t_{1}=486.82, t_{2}=-546.39, t_{3}=13777.0, b_{4}=61.5$, $x_{0}=0.83, x_{1}=-0.34, x_{2}=-1.00, x_{3}=1.35, \beta=\frac{1}{6}$. The values of $b_{i}$ and $b_{i}^{\prime}$ parameters in Eqs. (3) and (4) have been obtained from $t_{i}$ and $x_{i}$ by using the relations given in the Appendix A of Ref. [32]. We use two-body zero-range spin-orbit interaction by taking $b_{4}^{\prime}=b_{4}$ combination [27]. These parameters are the same as those given in Refs. [31, 32] except for the spin-orbit term which has been renormalized by taking $b_{4}=37.42$ so as to reproduce the single proton separation energy of ${ }^{8} \mathrm{~B}$. The adjusted values of the parameter $b_{4}$ is smaller than its original value of 61.50 . This observation is in line with the weakening 
of the spin-orbit interaction in light drip line nuclei noted in Ref. 34]. In the following the force with renormalized $b_{4}$ will be referred to as TH1 and that with the original $b_{4}$ as TH2.

With the same set of force parameters we have calculated total binding energies and root mean square (rms) radii of light unstable nuclei, ${ }^{8} \mathrm{Li},{ }^{8} \mathrm{~B},{ }^{7} \mathrm{~B},{ }^{7} \mathrm{Be}$, and ${ }^{9} \mathrm{C}$. Results for binding energies are presented in Table I where the corresponding experimental values are also shown [35]. Binding energies calculated with TH1 and TH2 forces are shown in third and fourth columns of this table, respectively. It is evident that with the renormalized $b_{4}$, our calculations reproduce the experimental binding energies of these nuclei to the extent of $98 \%$. Furthermore, the single neutron separation energy $\left(S_{n}\right)$ for ${ }^{8} \mathrm{~B}$ as calculated with the same force parameters is $13.47 \mathrm{MeV}$ which is in good agreement with the corresponding experimental value $13.02 \mathrm{MeV}$.

The rms radii for matter $\left(r_{m}\right)$, neutron $\left(r_{n}\right)$ and proton $\left(r_{p}\right)$ distributions are presented in Table II for all the five isotopes. Also shown in the first column of this table are corresponding experimental values of matter radii $\left(r_{m}^{e x p}\right)$ [7]. $r_{m}$ (shown in the second column) has been calculated by defining the total radius as the average of proton and neutron radii in every orbit weighted with occupation probabilities. $r_{p}$ and $r_{n}$ are shown in third and fourth columns, respectively of this table. We see that for all the isotopes $r_{m}$ calculated with the modified $b_{4}$ parameter, is in better agreement with the corresponding experimental data as compared to that calculated with the original $b_{4}$.

In Fig. 1, we show distributions of matter, charge, neutron and proton densities (in the units of $\mathrm{fm}^{-3}$ ) in the coordinate space. The nuclear charge density distribution has been obtained by folding the HF results for proton and neutron densities with the intrinsic charge density distribution of nucleons in Fourier-space by transforming the densities to formfactors. In actual calculations of the nuclear charge density, the center of mass correction effects are taken into account by unfolding the spurious vibrations of the nuclear center of mass in harmonic approximation (as is done in Eq. (7) for the zero-point energy). We note that nuclear charge and proton densities differ very slightly from each other. The key point of this figure is that the neutron and proton densities differ quite a bit from each other for distances larger than $3 \mathrm{fm}$, where the proton density develops a long tail. This is reminiscent of the situation in the neutron halo nuclei where the neutron density distribution has a long tail 2]. This observation supports the existence of a proton halo structure in ${ }^{8} \mathrm{~B}$. 


\section{B. Valence proton radius in ${ }^{8} B$ and Astrophysical $S_{17}$ factor}

To begin our discussions in this section we define the overlap function (OF)

$$
I_{7 B e-p}^{8 B}(\mathbf{r})=\left\langle\left[\psi_{7 B e}^{a s}\left(j_{x}\right) \otimes p_{j_{y}}\right]_{J M} \mid \psi_{8 B}^{a s}\left(j_{y}\right)\right\rangle_{J M}
$$

where symbol $\otimes$ denotes vector coupling. In Eq. (11), J is the total angular momentum which can be obtained by projecting the ground state wave function of ${ }^{8} \mathrm{~B}$ onto ${ }^{7} \mathrm{Be}$ ground state with spin-parity of $3 / 2^{-} . \quad \psi_{7_{B}}$ and $\psi_{8_{B}}$ are $\operatorname{SkHF}$ wave functions of ${ }^{7} \mathrm{Be}\left(p_{3 / 2}\right)$ and ${ }^{8} \mathrm{~B}\left(j_{y}\right)\left(j_{y}=3 / 2^{-}\right.$and $\left.1 / 2^{-}\right)$. These wave functions have been obtained with the same force parameters as those described in the previous section.

OF for ${ }^{8} B$ nucleus is used in the calculation of the asymptotic normalization constant which is related to the astrophysical factor $S_{17}$. It is also needed for the calculation of the valence proton density distribution which could be one more source of information about the existent of a proton halo structure in this nucleus. OF depends upon wave functions of ${ }^{8} \mathrm{~B}$ with given spin-parity combinations, which are completely antisymmetric in 'n' number active particles and the core state wave functions with the bound state having a total angular $\mathrm{J}$ and its projection $\mathrm{M}=0$. Due to the condition that the norm of $\mathrm{OF}\left(\left|I_{7 B e-p}^{8} B\right|\right)$ is always $\leq 1$, the overlap function is not a wave function of the total Hamiltonian.

The overlap function may be expressed in terms of the HF+BCS wave function as

$$
I_{7 B e-p}^{8 B}(\mathbf{r})=\sum_{J M}\left\langle j_{y} m_{y}, j_{x} m_{x} \mid J M\right\rangle Y_{l j_{y}, m_{y}}(\theta, \phi) I_{l j_{x}, j_{y}}^{8 B}(r) .
$$

The radial part of $\mathrm{OF}$ is written as,

$$
\left.I_{l j_{y}}^{8 B}(r)=\left.\sum_{j_{x}, j_{y}} v_{j_{x}} v_{j_{y}}\right|^{7} B e\left(j_{x}\right) \otimes p_{j_{y}}\right\rangle
$$

where $v_{j_{x}}$ and $v_{j_{y}}$ are the orbital occupancies of ${ }^{7} \mathrm{Be}$ and ${ }^{8} \mathrm{~B}$ nuclei, respectively.

The rms radius of the ${ }^{7} \mathrm{Be}+\mathrm{p}={ }^{8} \mathrm{~B}$ bound state (or the valence proton) can be written in terms of the overlap function as

$$
\left\langle r^{2}\right\rangle_{{ }_{B}}^{1 / 2}=\int_{0}^{\infty} r^{2} d r\left|I_{7 B e-p}^{8 B}(r)\right|
$$

The root mean square matter radius can be written in terms of $\left\langle r^{2}\right\rangle_{{ }_{B}}$ as

$$
<r_{m}^{2}>_{A}^{1 / 2}=\sqrt{\frac{1}{A+1}\left(A r_{c}^{2}+r_{p}^{2}+\frac{A}{A+1}\left\langle r^{2}\right\rangle_{{ }_{B}}\right)},
$$


where $\mathrm{A}$ is the mass of the core nucleus. In Eq. (15), $r_{c}$ is the rms matter radius of the core (its value is taken to be $2.33 \pm 0.02 \mathrm{fm}$ for ${ }^{7} \mathrm{Be}$ ), $r_{p}$ is the proton radius (taken to be $0.81 \mathrm{fm})$. This definition of the matter radius assumes that core and the valence particle are two distinct clusters and ignores the internal excitations of the core nucleus [36]. The rms matter radius calculated by Eq. (15) could be different from that discussed in the previous section even though HF wave functions have been used in both the calculations.

In table III, we show results of our calculations for $\left\langle r^{2}\right\rangle_{8_{B}}^{1 / 2}$ and $\left\langle r_{m}^{2}\right\rangle_{A}^{1 / 2}$. We have also shown in this table corresponding results of other authors who have used different models. We note that our results are in agreement with those of other authors within $10-20 \%$. Thus, almost all these calculations appear to be in agreement with the fact that the valence proton has a large spatial extension in ${ }^{8} \mathrm{~B}$. One exception, however, is Ref. [37] where a relatively smaller value of 3.75 fm has been reported for the valence proton rms radius in ${ }^{8} \mathrm{~B}$. These authors have done their calculations by employing a two-body model with a Gaussian potential of range $1.90 \mathrm{fm}$ which may not describe accurately the tail of the p-shell single proton wave function in ${ }^{8} \mathrm{~B}$.

We have estimated the contribution of the asymptotic part of the overlap wave function to the valence proton rms radius in ${ }^{8} \mathrm{~B}$ in the following way (see, e.g., 13]). We define the ratio

$$
C_{\nu}\left(R_{N}\right)=\left[\int_{R_{N}}^{\infty} r^{2 \nu} d r\left|I_{7 B e-p}^{8 B}(r)\right|^{2} / \int_{0}^{\infty} r^{2 \nu} d r\left|I_{7 B e-p}^{8 B}(r)\right|^{2}\right]^{1 / \nu}
$$

where $R_{N}$ is a cut-off radius. $\nu=1$ and 2 gives the contribution of the asymptotic part to the norm of the overlap function and the rms radius of the valence proton, respectively. For $R_{N}=2.5 \mathrm{fm}$ which is the calculated value of the matter radius in ${ }^{7} \mathrm{Be}$ (see table II), we find $C_{\nu}\left(R_{N}\right)=0.6260(0.8579)$. This indicates that the region out side the ${ }^{7} \mathrm{Be}$ core contributes up to $85 \%$ to the valence proton radius in ${ }^{8} \mathrm{~B}$ and that the probability of finding a valence proton outside the ${ }^{7}$ Be core nucleus is about $62 \%$. For $R_{N}=4.0 \mathrm{fm}$ (beyond which the nuclear interaction becomes negligible) we get $C_{1(2)}\left(R_{N}\right)=0.3248(0.5650)$ which means that contributions to the valence proton rms radius are about $56 \%$ from the distances beyond $4 \mathrm{fm}$. These results provide further support to the existence of a proton halo structure in ${ }^{8} \mathrm{~B}$ nucleus. As remarked earlier, in these calculations we have assumed that ${ }^{7}$ Be behaves as an inert core inside the ${ }^{8} \mathrm{~B}$ nucleus. Consideration of the excitation of ${ }^{7} \mathrm{Be}$ core will not change these conclusions significantly [13, 38].

It may be mentioned here that the experimental value of the quadrupole moment of ${ }^{8} \mathrm{~B}$ 
(which is twice as large as the value predicted by the shell model) can be explained with a single particle wave function corresponding to a matter radius of $2.72 \mathrm{fm}$ 7, 16]. This observation has been thought of as a possible evidence for a proton halo structure in ${ }^{8} \mathrm{~B}$. The matter radius of this nucleus as calculated by our model is very close to the above value.

Next, we present results for the astrophysical $S_{17}$ factor calculated within our model. In the region outside the core, where range of the nuclear interaction becomes negligible ( $\mathrm{r}>$ $4.0 \mathrm{fm})$, the radial overlap wave function $I_{l j}^{8} B(r)$ of the bound state can be written as

$$
I_{l j}^{8 B}(r) \simeq \bar{c}_{l j} W_{\eta, l+1 / 2}(k, r) / r
$$

where $W$ is the Whittaker function, $k$ the wave number corresponding to the single proton separation energy and $\eta$ the Sommerfeld parameter for the bound state in ${ }^{8} \mathrm{~B}$. In Eq. (17), $\overline{c_{l j}}$ is the asymptotic normalization constant, required to normalize the Whittaker function to the radial overlap wave function in the ${ }^{8} \mathrm{~B}$ nucleus in the asymptotic region. The $S_{17}$ factor is related to the proton capture cross section as

$$
S_{17}(E)=\sigma(E) E e^{(2 \pi \eta(E))} .
$$

It has been shown in ref. [19] that at low energies, the $S_{17}$ factor depends only on $\overline{c_{l j}}$ and one can write [24, 25]

$$
S_{17}^{A}=C_{N} \sum_{j_{y}} \bar{c}_{1, j_{y}}^{2}
$$

The value of $C_{N}$ depends on the details of the scattering wave function. In Ref. [24], $C_{N}=$ 37.8 has been obtained by using a microscopic cluster model for the scattering states, while a value of 36.5 has been reported for this quantity in Ref. [25] using a hard-core scattering state model.

In our calculations we have used our HF OF directly into a capture code where the scattering states are described by pure Coulomb wave functions between ${ }^{7}$ Be and $p$. Thus, within an inert ${ }^{7}$ Be core approximation our results are parameter free. In Table IV, we show $S_{17}^{A}$ obtained by using overlap functions calculated with both TH1 and TH2 force parameters. We see that $S_{17}^{A}$ obtained with TH1 force is quite close to its adopted limits of $19.1_{-1.0}^{+4.0} \mathrm{eV}$ b. On the other hand, that obtained with TH2 is quite large and well beyond the maximum limit of this value.

We also show in this table values of asymptotic normalization coefficients and $S_{17}^{A}$ obtained by using Eq. (19) with $C_{N}=36.5$ and 37.8. It is clear that there is a slight model dependence 
in the $S_{17}$ calculated in this way. These results however, are in agreement with those obtained by our method within $10 \%$.

Alternatively, $S_{17}$ factor can also be written in terms of the valence proton density around $10 \mathrm{fm}\left(\rho_{10}\right)$ and spectroscopic factors of valence proton states as [25]:

$$
S_{17}^{B}=2.99 \times 10^{6} \rho_{10} \times S_{\ell j}
$$

where $S_{\ell j}=S_{1,3 / 2}+S_{1,1 / 2}$ is the total spectroscopic factor, which may be calculated from the orbital occupancies. This relation has been used in Ref. [7] to calculate $S_{17}$ at the astrophysical energies from the shell model spectroscopic factors.

In case of ${ }^{8} \mathrm{~B}$, the last bound proton remains mostly in $p_{3 / 2}$ and $p_{1 / 2}$ orbitals. For the description of the dynamics of the valence proton with respect to the core nucleus in ${ }^{8} \mathrm{~B}$, we can use the one particle density operator. The radial dependence of the one body density operator, in the single particle operator approximation, can be written as:

$$
\rho_{j}(r)=\sum_{j m} a_{j m}^{\dagger}(r) a_{j m}(r)
$$

where $a^{\dagger}(r)[a(r)]$ is the creation (annihilation) operator for nucleons in the radial coordinate $r$ and in single particle state $|n l j m\rangle$. The above radial density can be transformed from particle operator representation to quasiparticle operator basis. This leads to the HF-BCS vacuum expectation value as,

$$
\rho(r)=\sum_{j} 2 \Omega_{j} v_{j}^{2} \phi_{j}^{2}(r)
$$

where $\Omega_{j}=j_{y}+1 / 2$ and $\phi(r)$ are radial single particle SkHF wave functions. Eq. (22) is the conventional expression for the one body radial density function in the quasiparticle representation.

In fig. 2, we show a comparison of the density distribution for the valence proton and that of protons in ${ }^{8} \mathrm{~B}$ and ${ }^{7} \mathrm{Be}$ nuclei. These densities are described above in terms of the quasi particle operator representation. As can be seen from this figure, the valence proton and ${ }^{8} \mathrm{~B}$ proton densities are similar for distances beyond the rms radius of ${ }^{7} \mathrm{Be}(2.50 \mathrm{fm})$. In order to exhibit clearly the comparison of densities at $10 \mathrm{fm}$, the density distributions are also shown on the linear scale in the region 9-11 $\mathrm{fm}$ in inset of this figure.

In table IV, we also show the theoretical results of spectroscopic factors (which can be calculated by integrating the square of the norm of quasi particle HF+BCS radial wave 
functions or from the orbital occupancies) and astrophysical $S_{17}$ factors obtained by using Eq (20) using both TH1 and TH2 force parameters. Values of the spectroscopic factors for $p_{3 / 2}$ and $p_{1 / 2}$ orbitals do not differ significantly from each other in the two cases. However, the valence proton density at $10 \mathrm{fm}$ is lower in SkHF with TH2 force case by a factor of about 2 as compared to that with TH1 force. This leads to a considerable lower value for the $S_{17}$ factor in the former case. With TH1 density at $10 \mathrm{fm}$ we get the astrophysical $S_{17}$ factor of $19.7 \mathrm{eV}$ b which is about the same as that obtained above. However, in the potential model calculations Ref. 25] the difference between $S_{17}^{A}$ and $S_{17}^{B}$ is quite large.

\section{SUMMARY AND CONCLUSIONS}

In summary, in this paper we studied the structure of ${ }^{8} \mathrm{Li},{ }^{8} \mathrm{~B},{ }^{7} \mathrm{~B},{ }^{7} \mathrm{Be}$, and ${ }^{9} \mathrm{C}$ nuclei within the Skyrme Hartree-Fock framework with SLy4 interaction parameters whose spinorbit part is renormalized so as to reproduce the last proton binding energy in ${ }^{8} \mathrm{~B}$. The adjusted spin orbit term is weaker than that of the original force. We calculated binding energies, various densities distribution and rms radii for these nuclei. Using the same set of the force parameters, we obtain good agreements with experimental values of binding energies and rms matter radii for all these nuclei. We have calculated the overlap function $<\left.^{7} \mathrm{Be}\right|^{8} \mathrm{~B}>$ from the SkHF wave functions which has been employed to obtain the radius of the valence proton in ${ }^{8} \mathrm{~B}$ nucleus. The value of this quantity is found to be $4.93 \mathrm{fm}$ which is almost two times larger than the matter radius of ${ }^{7} \mathrm{Be}$ core. This provides support to the possibility of ${ }^{8} \mathrm{~B}$ having a one proton halo structure.

The same overlap function is used to extract the asymptotic normalization coefficients for ${ }^{8} \mathrm{~B} \rightarrow{ }^{7} \mathrm{Be}+p$. Using the overlap function calculated with the modified force we obtain an astrophysical S-factor of $22.0 \mathrm{eV}$ b while the original parameterization leads to a value of $35.0 \mathrm{eV}$ b. Thus the $S_{17}$ calculated with the TH1 force lies within the adopted limits $\left(19.1_{-1.0}^{+4.0} \mathrm{eV}\right.$ b) of the near zero energy astrophysical S-factor. The values of $S_{17}$ obtained by using the corresponding asymptotic normalization coefficients follow the similar trend. Although some dependence on the scattering model used in these calculations is apparent. We also calculated $S_{17}$ from the valence proton density in the asymptotic region and the spectroscopic factors of the p-orbitals in ${ }^{8} \mathrm{~B}$. The value of the $S_{17}$ factor obtained in this way is $19.7 \mathrm{eV}$ b, which is quite close to the value obtained from the overlap function. The 
average of these two values is 21.1 eV.b.

The results of our calculations have a strong dependence on the parameter of the spinorbit term of the Skyrme interaction. This suggests that it may be possible to have some important clue about the effective interaction in drip line nuclei from the comparison of calculations with some experimental observables.

\section{ACKNOWLEDGMENT}

This work is supported by the Department of Atomic Energy, (BRNS), (BARC), Mumbai, under contract no. 2001/37/14/BRNS/699.

[1] I. Tanihata et al., Phys. Rev. Lett. 55, 2676 (1985); I. Tanihata, Prog. Part. Nucl. Phys. Vol. 35, 505 (1995); A. Ozawa, T. Kobayashi, T. Suzuki, K. Yoshida and I.Tanihata, Phys. Rev. Lett. 84, 5493 (2000).

[2] P.G. Hansen, A.S. Jensen and B. Jonson, Annu. Rev. Nucl. Part. Sci. 45591 (1995).

[3] J.N. Bachcall Neutrino Astrophysics (Cambridge University Press, 1989), J.N. Bachall and M. Pinsonneault, Rev. Mod. Phys. 64, 885 (1992).

[4] R. Davis, Prog. Part. Nucl. Phys. 32, 13(1994), Eric G. Adelberger et al., Rev. Mod. Phys. 70, 1265 (1998).

[5] S. Fukuda et al, Phys. Rev. Lett. 86, 5651 (2001), Q.R. Ahmad et al., 87, 071031 (2001), 89, 011301 (2002).

[6] I. Tanihata,T. Kobayashi, O. Yamakawa, S. Shimoura, K. Ekuni, K. Sugimoto, N. Takashi, T. Shimoda and H. Sato, Phys. Lett. 206B 592 (1988).

[7] F. Negoita et al., Phys. Rev. C54, 1787 (1996).

[8] B. Blank et al., Nucl. Phys. A624, 242 (1997).

[9] H. Esbensen and K. Hencken, Phys. Rev. C61, 054606 (2000).

[10] T. Barker et al., Phys. Rep. 289, 235 (1997).

[11] R. Shyam and H. Lenske, Phys. Rev. C57, 2427( 1998).

[12] N.K. Timofeyuk, Nucl. Phys. A632, 19 (1998). 
[13] F. Carstoiu, L. Trache, C.A. Gagliardi, R.E. Tribble and A.M. Mukhamedzhanov, Phys. Rev. C63, 054310 (2001).

[14] M.H. Simedberg et al., Phys. Lett. B452, 1 (1999).

[15] L.V. Grigorenko, B.V. Danilin, V.D. Efros, N.B. Shulgina and M.M. Zhukov, Phys. Rev.C57, R2099 (1998).

[16] M.M. Obut et al., Nucl. Phys. A609, 74 (1996), R.E. Warner, et al., Phys. Rev. C 52, R1166 (1995).

[17] B.W. Fillippone, S.J. Elwyn, C.N. Davids, D.D. Koetke, Phys. Rev. Lett. 50, 412 (1983); Phys. Rev. C28, 2222 (1983); F. Hammache et al., Phys. Rev. Lett 80, 928 (1998); A.R. Junghans et al., Phys. Rev. Lett. 88, 041101 (2002).

[18] T. Motobayashi et al., Phys. Rev. Lett. 73, 2680 (1994); I. Motobayashi, Nucl. Phys. A693, 258 (2001); N. Iwasa et al., Phys. Rev. Lett. 83, 2910 (1999); B. Davids, et al., Phys. Rev. C63, 065806 (2001).

[19] H.M. Xu, C.A. Gagliardi, R.E. Tribble, A.M. Mukhamedzhanov and N.K. Timofeyuk, Phys. Rev. Lett. 73, 2027 (1994).

[20] G.A. Gagliardi et al., Nucl. Phys. A682, 369 (2001).

[21] A. Azhari et al., Phys. Rev. C60, 055803 (1999).

[22] W. Lui et al., Nucl. Phys. A616 131c (1997); Phys. Rev. Lett 77, 611 (1996).

[23] N.K. Timofeyuk, D. Baye, and P. Descouvemint, Nucl. Phys. A620, 29 (1997).

[24] A. Csoto, Phys. Rev. C61, 037601 (2000); B.K. Jennings, S. Karataglidis, and T.D. Shoppa, Phys. Rev. C 58, 3711 (1998).

[25] B.A. Brown, A. Costo, and R. Sherr, Nucl. Phys. A597, 66 (1996).

[26] J.W.Negele and D. Vautherin, Phys. Rev. C5, 1472 (1972).

[27] J. Friedrich and P.-G. Rienhard, Phys. Rev. C33, 335 (1986).

[28] J. Dobaczewski, I. Hamamoto, W. Nazarewicz and J.A. Sheikh, Phys. Rev. Lett. 72, 981 (1994).

[29] Yao-song Shen and Zhogzhon Ren, Phys. Rev. C54, 1158 (1996).

[30] X. Li and P.-H. Heensen, Phys. Rev. C54, 1617 (1996).

[31] S. Mizutori, J. Dobaczewski, G.A. Lalazissis, W. Nazarewicz and P.-G. Reinhard, Phys. Rev. C61, 044326 (2000).

[32] P.-G. Reinhard, D.J. Dean, W. Nazarewicz, J. Dobaczewski, J.A. Maruhn and M.R. Strayer, 
Phys. Rev. C60, 014316 (1999).

[33] E. Chabanat, P. Bonche, P. Haensel, J. Meyey and R. Schaeffer, Nucl. Phys. A.627, 710(1997).

[34] G. A. Lalazissis, D. Vretenar, W. Pöschl, and P. Ring, Phys. Lett. B418, 7 (1998).

[35] R.B. Firestone, V.S. Shirley, C.M. Bagln, S.Y.E. Chu and J. Zipkin, Table of Isotopes ( Wily, New York 1996).

[36] B. Buck and A.A Pilt, Nucl. Phys. A280, 133 (1977).

[37] K. Riisager and J.S. Jensen, Phys. Lett. B301, 6 (1993).

[38] A.M. Moro, R. Crespo, F. Nunes and I.J. Thompson, Phys. Rev. C66, 024612 (2002). 
TABLE I: The comparison of theoretical binding energies for various nuclei calculated in selfconsistent SkHF method with experimental data. The TH1 and TH2 represent theoretical results obtained with modified and original values of the $\left(b_{4}\right)$ parameter of the SLy4 Skyrme force, respectively.

\begin{tabular}{cccr}
\hline \hline Nucleus & & BE $(\mathrm{MeV})$ & \\
\hline & Expt. & & Theor. \\
& & TH1 & TH2 \\
\hline${ }^{7} B e$ & 37.601 & 37.561 & 39.780 \\
${ }^{7} B$ & 24.720 & 24.262 & 25.931 \\
${ }^{8} \mathrm{Li}$ & 41.278 & 41.034 & 44.098 \\
${ }^{8} \mathrm{~B}$ & 37.739 & 37.739 & 40.677 \\
${ }^{9} \mathrm{C}$ & 39.716 & 39.035 & 37.416 \\
\hline \hline
\end{tabular}

TABLE II: Rms mass $\left(r_{m}\right)$, proton $\left(r_{p}\right)$ and neutron $\left(r_{n}\right)$ radii for various nuclei. The TH1 and TH2 represents the theoretical results obtained with modified and original values of the $\left(b_{4}\right)$ parameter of the SLy4 Skyrme force, respectively. The experimental values of rms radii in ${ }^{7} \mathrm{Be}[6]$, ${ }^{8} \mathrm{Li}[16],{ }^{8} \mathrm{~B}[7,16]$ and ${ }^{9} \mathrm{C}[38]$ are also shown here.

\begin{tabular}{|c|c|c|c|c|c|c|c|c|c|}
\hline \multirow[t]{4}{*}{ Nucl. } & \multicolumn{9}{|c|}{$\operatorname{rms} \operatorname{radii}(\mathrm{fm})$} \\
\hline & \multirow{2}{*}{\multicolumn{3}{|c|}{ Expt. }} & \multicolumn{6}{|c|}{ Theor. } \\
\hline & & & & \multicolumn{3}{|c|}{ TH1 } & \multicolumn{3}{|c|}{ TH2 } \\
\hline & $r_{m}^{e x p}$ & $r_{p}$ & $r_{n}$ & $r_{m}$ & $r_{p}$ & $r_{n}$ & $r_{m}$ & $r_{p}$ & $r_{n}$ \\
\hline${ }^{7} B e$ & $2.33 \pm 0.02$ & - & - & 2.49 & 2.63 & 2.29 & 2.32 & 2.46 & 2.12 \\
\hline${ }^{7} B$ & - & - & - & 2.86 & 3.18 & 1.84 & 2.73 & 3.01 & 1.87 \\
\hline${ }^{8} \mathrm{Li}$ & $2.37 \pm 0.02$ & $2.26 \pm 0.02$ & $2.44 \pm 0.02$ & 2.54 & 2.29 & 2.67 & 3.01 & 2.98 & 3.02 \\
\hline \multirow[t]{2}{*}{${ }^{8} B$} & $2.55 \pm 0.08$ & $2.76 \pm 0.08$ & $2.16 \pm 0.08$ & 2.57 & 2.73 & 2.27 & 2.84 & 2.96 & 2.73 \\
\hline & $2.43 \pm 0.03$ & $2.49 \pm 0.03$ & $2.33 \pm 0.03$ & & & & & & \\
\hline${ }^{9} C$ & $2.42 \pm 0.03$ & & & 2.59 & 2.77 & 2.20 & 2.13 & 2.32 & 1.67 \\
\hline
\end{tabular}


TABLE III: SkHF results for the root-mean-square radius $<r_{8_{B}}^{2}>^{1 / 2}$ for the ${ }^{7} \mathrm{Be}+\mathrm{p}={ }^{8} \mathrm{~B}$ bound state and rms matter radius $<r_{m}^{2}>^{1 / 2}$ in unit of $\mathrm{fm}$.

\begin{tabular}{cccc}
\hline \hline MODEL(ref.) & $\left\langle r_{8_{B}}^{2}\right\rangle^{1 / 2}$ & $\left\langle r_{m}^{2}\right\rangle_{A}^{1 / 2}$ & Ref. \\
\hline SkHF & & & present work \\
RPA+Mean-field & 4.93 & 2.74 & {$[10,11]$} \\
Microscopic & 4.73 & 2.70 & {$[12]$} \\
many-body & 4.43 & 2.68 & \\
calculation & & & {$[37]$} \\
Two-body & 3.75 & & \\
Model & & 2.52 & {$[15]$} \\
Cluster Model & - & $2.50 \pm 0.04$ & {$[13]$} \\
ANC method & $4.20 \pm 0.22$ & $2.60 \pm 0.04$ & {$[8,9]$} \\
Exp. & $4.64 \pm 0.23$ & $2.83 \pm 0.06$ & {$[7]$} \\
Exp. & $3.97 \pm 0.12$ & $2.55 \pm 0.18$ & {$[14]$} \\
Exp. & $4.22-4.50$ & $2.58-2.60$ & {$[16]$} \\
Exp. & - & 2.72 & \\
\hline \hline
\end{tabular}


TABLE IV: SkHF results for asymptotic normalization coefficients $\left(\bar{c}_{l, j}\right)$, spectroscopic factors $\left(S_{l j_{y}}\right)$, densities of valence particle $(\rho)$ at $10 \mathrm{fm}$ radius, and astrophysical $S$ - factors $S_{17}^{A}$ and $S_{17}^{B}$. $S_{17}^{A 1}$ corresponds to results obtained by using the SkHF overlap function directly to the capture code while $S_{17}^{A 2}$ and $S_{17}^{A 3}$ to those obtained by using Eq. (19) with $C_{N}=36.5$ and 37.8 , respectively.

\begin{tabular}{ccc}
\hline \hline & & SkHF \\
& TH1 & TH2 \\
\hline $\bar{c}_{1,3 / 2}$ & 0.64 & 0.88 \\
$\bar{c}_{1,1 / 2}$ & 0.34 & 0.29 \\
$S_{17}^{A 1}(\mathrm{eVb})$ & 22.0 & 35.3 \\
$S_{17}^{A 2}(\mathrm{eVb})$ & 19.5 & 31.3 \\
$S_{17}^{A 3}(\mathrm{eVb})$ & 20.2 & 32.4 \\
& & \\
\hline$S_{0 p_{3 / 2}}$ & 1.02 & 0.95 \\
$S_{0 p_{1 / 2}}$ & 0.42 & 0.45 \\
$\rho(10 \mathrm{fm}) \times 10^{-6}$ & 4.58 & 11.9 \\
$S_{17}^{B}(e V b)$ & 19.7 & \\
\hline \hline
\end{tabular}




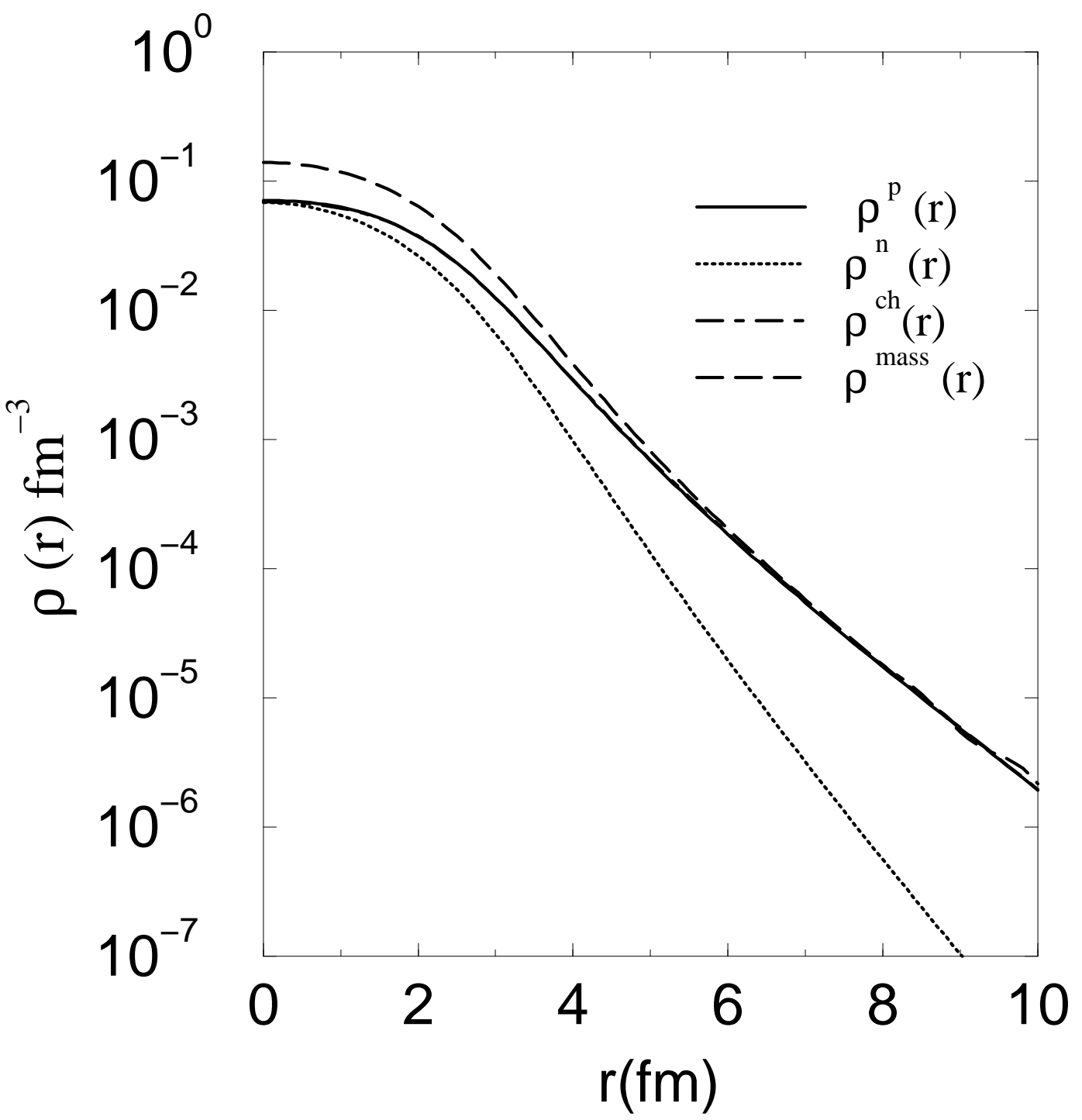

FIG. 1: Density distribution $\rho(r)$ for protons, neutrons, nuclear charge and mass in ${ }^{8} B$ nucleus calculated with SkHF method. 


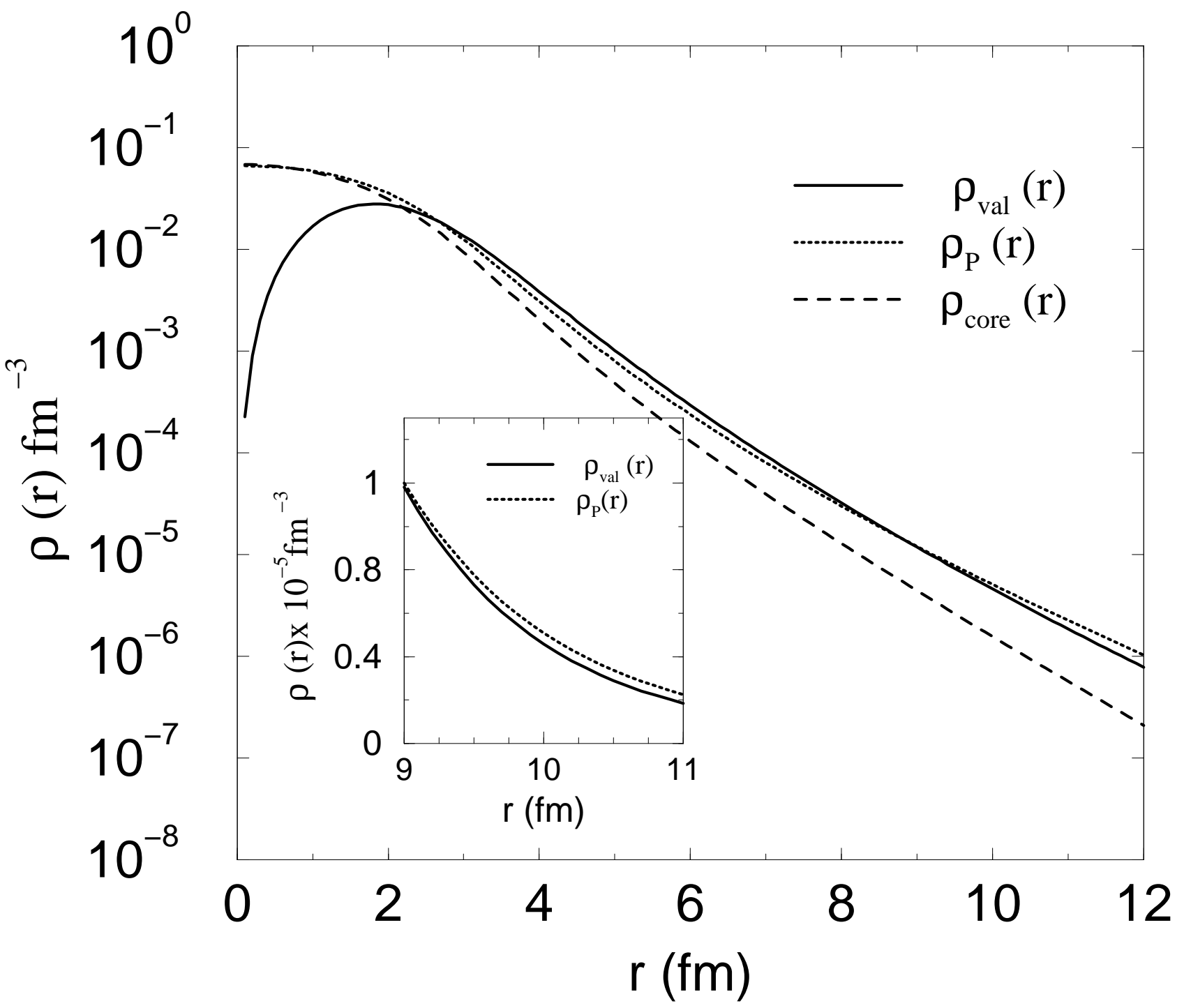

FIG. 2: The valence proton density $\rho_{\text {val }}(r)$ and the proton density $\rho_{p}(r)$ in ${ }^{8} B$ as a function of the radial coordiante. Also shown is the r-space distribution of the density of core nucleus, $\rho_{\text {core }}(r)$. 